\title{
ESTUDO ANATÔMICO DAS VARIAÇÕES DE POSIÇÃO DA JUNÇÃO DOS DUCTOS CÍSTICO E HEPÁTICO COMUM EM FETOS ${ }^{1}$
}

\author{
Michael Silveira Santiago ${ }^{2}$ \\ Tatiana Silveira Santiago ${ }^{3}$ \\ Valdinaldo Aragão de Melo $^{4}$ \\ Jorge Carvalho Mendonça ${ }^{5}$
}

\begin{abstract}
Santiago MS, Santiago TS, Melo VA, Mendonça JC. Estudo anatômico das variações de posição da junção dos ductos cístico e hepático comum em fetos. Acta Cir Bras [serial online] 2003 JanFev;18(1). Disponível em URL: http://www.scielo.br/acb.

RESUMO - Objetivo: Definir e classificar as variações anatômicas da junção dos ductos cístico e hepático comum em fetos, analisando a freqüência, trajeto e relação entre eles. Métodos: Dissecaramse 33 fetos, no período de setembro de 1999 a julho de 2000, utilizando-se fotografias para registrar as junções cístico-hepáticas. As uniões foram classificadas como alta, média e baixa e, quanto ao curso, paralelo ou angular. Constatado o tipo de união, aferiu-se o comprimento dos ductos. Resultados: Visualizou-se a junção cístico-hepática em $93,9 \%$ dos fetos, sendo encontrada a inserção média em $45,2 \%$ delas, a inserção alta em $29 \%$ e a inserção baixa em $25,8 \%$. Quanto ao curso ductal, a união aguda foi observada em $71 \%$ dos fetos, enquanto a paralela, em $29 \%$. Quanto ao comprimento ductal, o ducto cístico variou de $4-6 \mathrm{~mm}$, o ducto hepático comum de 9 - 13 $\mathrm{mm}$, e o ducto colédoco de $5-10 \mathrm{~mm}$. Conclusão: Dentre as variações anatômicas, a inserção média foi a prevalente, seguida do curso angular, com comprimentos dos ductos cístico e hepático comum variando entre $6 \mathrm{~mm}$ e $7 \mathrm{~mm}$, respectivamente. Foi demonstrada uma freqüência significativa de inserção baixa cístico-hepático comum.
\end{abstract}

DESCRITORES - Ducto cístico. Ducto hepático. Anatomia.

\section{INTRODUÇÃO}

A anatomia da árvore biliar extra-hepática é de grande relevância na cirurgia biliar, principalmente no que concerne às variações das junções dos ductos cístico e hepático comum $(\mathrm{C}-\mathrm{H})$, que vão se configurando ao longo do desenvolvimento embriológico do indivíduo ${ }^{1-8}$.
De acordo com o tipo de junção C-H encontrado, pode haver maior ou menor risco de lesão destas estruturas durante intervenções cirúrgicas ou na evolução de processos patológicos ${ }^{9}$.

A classificação dessa junção é descrita segundo o curso dos ductos $\mathrm{C}-\mathrm{H}$, que pode ocorrer em paralelo ou angular. A depender da distância da ampola duodenal à

1. Trabalho realizado no laboratório de Anatomia do Departamento de Morfologia da Faculdade de Medicina na Universidade Federal de Sergipe (UFS).

2. Acadêmico do $6^{\circ}$ ano do Curso de Medicina da Universidade Federal de Sergipe

3. Acadêmica do $6^{\circ}$ ano do Curso de Medicina da Universidade Federal de Sergipe.

4. Professor Adjunto de Cirurgia do Aparelho Digestivo do Departamento de Medicina da Universidade Federal de Sergipe.

5. Professor Titular de Anatomia Humana I do Departamento de Morfologia da Universidade Federal de Sergipe. 
bifurcação do ducto hepático comum, ela será designada como alta (proximal), média e baixa (distal) ${ }^{10-12}$.

A variabilidade anatômica da árvore biliar compreende principalmente as anomalias do sistema ductal, permitindo que várias situações morfológicas variantes comprometam a fisiologia hepatobiliar ${ }^{13,14}$.

Este trabalho tem como objetivo classificar as junções $\mathrm{C}-\mathrm{H}$, mediante sua visualização e reconhecimento da posição e disposição dos ductos biliares em fetos, comparando os achados com os relatos da literatura, além de descrever a presença de anomalias dos referidos ductos ${ }^{15-18}$.

\section{MÉTODOS}

Foram analisados, no período compreendido entre setembro de 1999 a julho de 2000, 33 natimortos procedentes do laboratório de anatomia do Departamento de Morfologia da Universidade Federal de Sergipe, obtidos da Maternidade Hildete Falcão Batista, após consentimento informado das parturientes. Os processos metodológicos realizados foram feitos na seguinte seqüência: 1 - Processo de obtenção dos ductos: o fígado foi retirado em bloco juntamente com a vesícula biliar e a segunda porção do duodeno, a fim de preservar os ductos cístico e hepático comum e o ducto colédoco. O material foi conservado em formol a 10\%. 2 - Processo de visualização dos ductos: os ductos cístico, hepático comum e colédoco, que não apresentaram processos patológicos, foram dissecados em condições naturais ou com auxílio de lupa. Os ductos não receberam qualquer tipo de tratamento prévio para seu preenchimento. 3 - Dados anatômicos - Avaliação dos tipos de inserção, curso e comprimento ductais segundo a classificação de Shaw et al. (1993). As peças foram documentadas fotograficamente e os dados foram tabelados e comparados com os descritos na literatura.

\section{RESULTADOS}

A junção cístico-hepático comum foi observada em 31 fetos $(93,9 \%)$ dos 33 dissecados. Desta amostra, obtivemos os seguintes dados quanto ao tipo de inserção, curso e cumprimento ductais:

- A inserção média foi encontrada em 14 (45,2\%) das junções visualizadas (Figura 1); a inserção alta foi observada em 9 (29,0\%), e a inserção baixa em 8 , correspondendo a $25,8 \%$ (Figura 2)

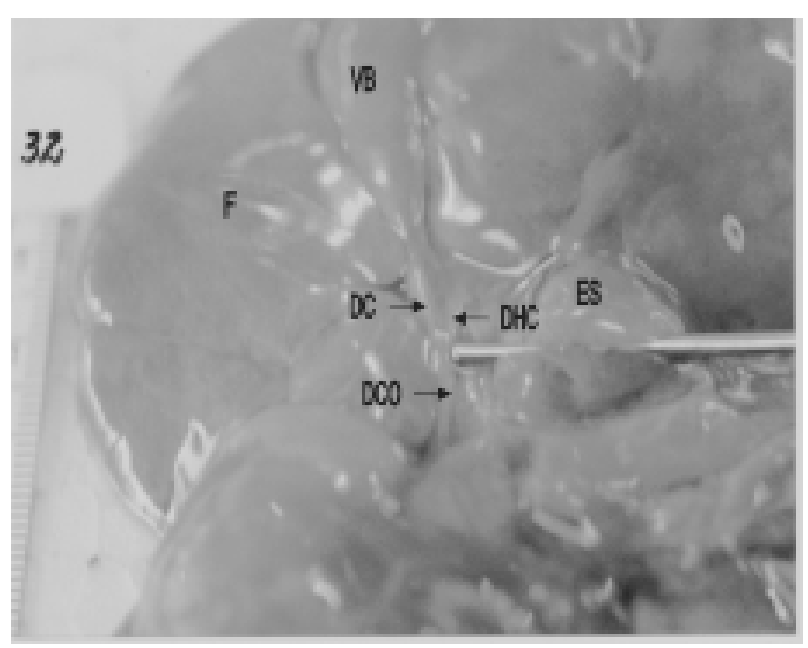

FIGURA 1 - Inserção média com curso dos DC e DHC (feto no. 32) VB (vesícula biliar); F (fígado); DC (ducto cístico); DHC (ducto hepático comum); DCO (ducto colédoco); ES (estômago).

\section{Porcentagem de Tipos de Inserções por Fetos}
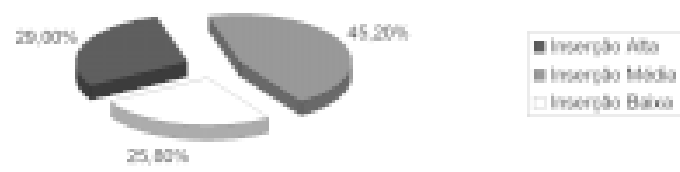

FIGURA 2 - Classificação angular segundo o tipo de inserção.

Quanto à classificação em angular e paralela, registrou-se que a união angular estava presente em 22 $(71,0 \%)$. Não foi possível caracterizar o curso dos ductos em espiral, pois grande parte da amostra foi estudada a fresco, o que alterava a configuração espacial das peças, que é observada quando in vivo (Figura 3).

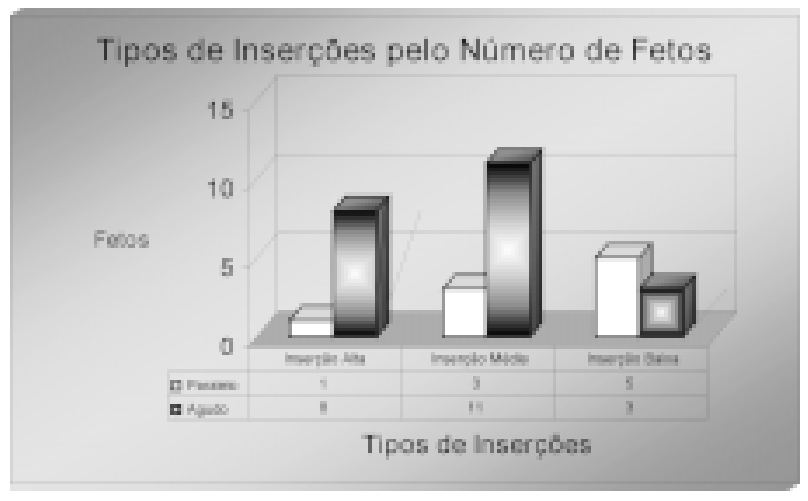

FIGURA 3 - Relação entre os tipos de cursos dos ductos cístico e hepático comum até sua união com o tipo de inserção dos mesmos. 
Em relação ao comprimento dos ductos, observou-se que a mensuração variava de acordo com o tipo se inserção (alta, média ou baixa). Logo, quando a inserção era alta, o ducto cístico (DC) variava de 4$6 \mathrm{~mm}$ e ducto hepático comum (DHC) de 3-9mm. Contudo, quando a inserção era baixa, o comprimento do DC oscilava de $9-13 \mathrm{~mm}$ e do DHC de $7-9 \mathrm{~mm}$. No que se refere ao comprimento dos ductos quando em inserção média, o DC media de 5-10mm e o DHC de 4$8 \mathrm{~mm}$. As médias obtidas dos ductos cístico e hepático comum foram de $6 \mathrm{~mm}$ e $7 \mathrm{~mm}$, respectivamente.

\section{DISCUSSÃO}

O estudo da anatomia dos trajetos dos ductos da árvore biliar extra-hepática é fundamental para a evolução da técnica cirúrgica, assim como para o melhor conhecimento de patologias que acometem esta região. Diversos estudos foram feitos com este intuito, utilizando-se cadáveres ou através de estudos intraoperatórios, $\mathrm{e}$, à medida que mais pesquisas vão sendo desenvolvidas neste sentido, aumentam o número de anomalias relatadas.

Hand, em 1973, reconheceu as três formas mais comuns de entrada do ducto cístico, onde a mais freqüente era a angulação lateral, que ocorria em $65 \%$ dos casos. Em seguida, o curso paralelo, que ocorria em $25 \%$ dos casos (ducto cístico cursando paralelo em relação ao DHC). E, por último, o curso ductal em espiral, que ocorria em $10 \%$ dos casos.

Foram encontrados sistemas de ductos paralelos em $29 \%$ dos fetos estudados, variando em relação ao valor encontrado por Shaw et al. (1993). Entretanto, apenas um caso $(3,22 \%)$ foi relatado de aderência completa do ducto cístico com o hepático comum. Em relação aos dados da pesquisa de Shaw, este classifica o DC em três tipos de orientação radial, podendo ser lateral, medial e espiral, de modo que a mais freqüente é a lateral ( $50 \%$ da amostra), e a menos encontrada, a medial. Observou-se neste trabalho, uma concordância com nossos resultados, pois a maioria dos fetos apresentava orientação lateral do ducto cístico.

Embora se encontrem variações anatômicas dos $\mathrm{DHC}$ e DC com relativa freqüência na literatura, não as observamos neste trabalho. $\mathrm{O}$ que contribui para o relato dos achados variantes é a utilização de técnicas de imagem não invasivas ou feitas no intraoperatório (durante colecistectomias), realizadas para se obter manejo mais seguro das estruturas da região hepatobiliar $^{9,13,19,20,21}$.

É importante frisar que há alguns fatores de risco, dentre as variantes anatômicas, para maior incidência de lesão ductal. De acordo com Taourel et al. (1996), constituem-se de: 1. Presença de inserção aberrante do ducto hepático direito no ducto hepático comum ou no ducto cístico; 2. Inserção baixa do ducto cístico no ducto hepático comum; 3. Curso paralelo dos ductos cístico e hepático; 4. Curso espiral do ducto cístico; 5. Ducto cístico curto. Em vista disso, procurou-se dedicar maior enfoque às inserções baixas e ao curso paralelo, pois foram os fatores de risco de maior incidência neste trabalho.

Deve-se considerar que algumas divergências nos resultados encontrados nesta pesquisa e os da literatura, podem ser devido ao pequeno número de fetos abordados, o que também não exclui a possibilidade de variações dos dados a depender da população estudada.

\section{CONCLUSÃO}

Este trabalho revela-se de grande significância por ter sido realizado em fetos. Dentre as variações de inserção ductal, a inserção média foi a mais prevalente, e quanto ao curso ductal, o angular foi o mais freqüente. A inserção ductal baixa foi a de menor incidência, porém é a que merece maior atenção, pelo risco de lesão. O conhecimento anatômico dessas estruturas é de fundamental importância para que se possa evitar iatrogenia em cirurgia.

\section{REFERÊNCIAS}

1. Gray H. Anatomia. 29ed. Rio de Janeiro: Guanabara Koogan 1988 .

2. Haga T, Uchimura F. Cystic duct anatomy on DIC-helical CT. Nippon Igaku Hoshasen Gakkai Zasshi 1995;55(11):776-8.

3. Hand $\mathrm{BH}$. Anatomy and function of the estrahepatic biliary system. Clin Gastroenterol 1973;2(1):3-29.

4. Hofer Pineda U, Hofer Hunro W, Navarrete Ventura M. Vías biliares extrahepáticas en fetos humanos de término Extrahepatic biliary duct in term human fetus. An Anat Norm 1984;2(2):62-6.

5. Healy JE, Schoroy PG. Anatomy of the biliary ducts within the human liver. Analysis of the prevailing pattern of btanchungs and the major variations of the biliary ducts. Arch Surg 1953;66:599-616

6. Hollinshead W H. Anatomy For Surgeons. 2ed. New York: Harper E Row; 1971

7. Junqueira V. Fundamentos de Embriologia Humana. 2ed. Rio de Janeiro: Guanabara Koogan; 1977. Beaver MG. Variations in the extrahepatic biliary tract. Arch Surg 1929;19:321-6.

8. Testut L, Latarjet A. Tratado de Anatomia Humana. 9ed. Barcelona: Salvat; 1968

9. Taourel P, Bret PM, Reinhod C, Barkun NA, Atri M. Anatomic variants of the biliary tree: diagnosis with $M R$ cholangiopancreatography. Radiology 1996;199(2):521-7.

10. Köhler R, Viljanev V. Spiral tipe juction of the cystic and common hepatic ducts investigated by operative cholangiography. Acta Chir Scand 1963;126:621.

11. Uetsuji S, Okuda Y, Komada H, Yamamura M, Kamiyama Y. Clinical evaluation of a low junction of the cystic duct. Scand J Gastroenterol 1993;28(1):85-8 
12. Yoshida J, Chijiiwa K, Yamaguchi K, Yokohata K, Tanaka M Practical classification of the branching types of the biliary tree: an analysis of 1094 consecutive direct cholangiograms. J Am Coll Surg 1996;182(1):37-40.

13. Beaver MG. Variations in the extrahepatic biliary tract. Arch Surg 1929;19:321-6.

14. Tsunoda M, Akaki S, Togami I, Hiraki Y. Anatomic variants of the cystic duct and cysticohepatic junction: diagnosis with MR cholangiography. Nippon Rinsho 1998;56(11):2918-22.

15. Eisendrath DN. Anomalies of the bile ducts and blood vessels: As the cause of accidents in biliary. JAMA 1918;71:864.

16. Fujikawa T, Takeda H, Matsusue S, Nakamura Y, Nishimura S. Anomalous duplicated cystic duct as a surgical hazard: report of a case. Surg Today 1998;28(3):313-5.

17. Langley JR, Rosato FE. Anomalies of the biliary duct system. Va Med Mon 1976;103(10):734-5.
18. Shaw MJ, Dorsher PJ, Vennes JA. Cystic duct anatomy: an endoscopic perspective. Am J Gastroenterol $1993 ; 88(12): 2102-6$

19. Losanoff JE, Kjossev KT, Katrov E. Hepaticocystic duct- a case report. Surg Radiol Anat 1996;18(4):339-41.

20. Stemple JF. The need for careful operative dissection in Moosman's area during cholecystectomy. Surg Gynecol Obstet 1986;163(2):169-73.

21. Todani T, Watanabe Y, Toki A, Ogura K, Wang ZQ. Co-existing biliary anomalies and anatomical variants in choledochal cyst. Br J Surg 1998;85(6):760-3.

\section{AGRADECIMENTO}

A Ana Ramalho, por sua contribuição na documentação fotográfica dos dados desta pesquisa.

Santiago MS, Santiago TS, Melo VA, Mendonça JC. Anatomical variability of the junction between cystic and common hepatic ducts in fetus. Acta Cir Bras [serial online] $2003 \mathrm{Jan}-\mathrm{Feb} ; 18(1)$. Available from URL: http://www.scielo.br/acb.

ABSTRACT - Purpose: To define and to classify the anatomical variability of the junction between cystic and common hepatic ducts in fetus, it was studied the frequency, itinerary and relationship among them, emphasizing their anastomoses. Methods: From 1999 to 2000, 33 fetus were dissected. Using pictures to register the cysticohepatic junctions, they were classified as high, medial and low, and the course type in parallel or angular. Results: The cysticohepatic junction was visualized in $93,9 \%$ of the fetus, the medial insert in $45,2 \%$ of them, the high insert in $29,0 \%$ and the low one in $25,8 \%$. Concerning the ductal course, the angular union was present in $71 \%$ of the fetus, while the parallel was in $29 \%$. About the length of the ducts, the cystic duct varied from 4 to $6 \mathrm{~mm}$, the common hepatic duct from 9 to $13 \mathrm{~mm}$ and the choledochal duct from 5 to $10 \mathrm{~mm}$. Conclusion: Concerning anatomic variations, the medial insertion was prevalent associated with angular course. Mean length of the cystic and common hepatic ducts were 6 and $7 \mathrm{~mm}$, respectively. This study demonstrated a significant frequency of cysticohepatic low junctions.

KEY WORDS - Cystic ductal. Hepatic duct. Anatomy.

Conflito de interesse: nenhum

Fonte de financiamento: $\mathrm{CNPq}$

Correspondência:

Rua Álvaro Brito, 14/301

49020-400 Aracaju - SE

vamelo@infonet.com.br

Data do recebimento: 22/09/2002

Data da revisão: 11/10/2002

Data da aprovação: 19/10/2002 